\title{
Carles Puigdemont und die Gretchenfrage der Immunität
}

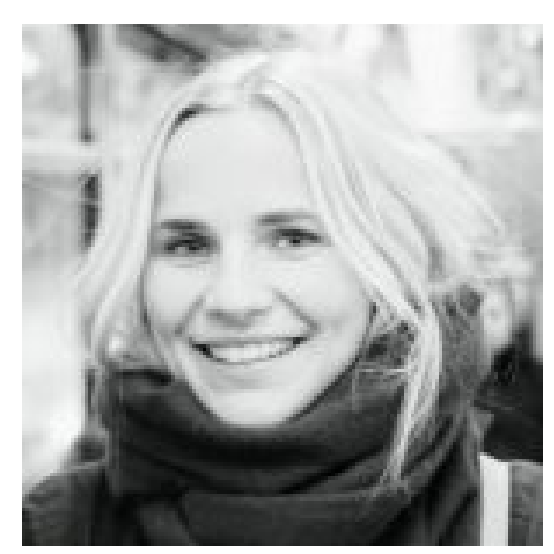

FREDERIKE FRÜND

von

Am 2. Juli 2019 findet die konstituierende Sitzung des Europäischen Parlaments in Brüssel statt. Offen ist die Frage, ob der ehemalige katalanische Regionalpräsident Carles Puigdemont an dieser Sitzung teilnehmen wird. Nach spanischem Wahlrecht kann er nur dann als EU-Abgeordneter bestätigt werden, wenn er in Madrid seine Abgeordnetenurkunde abholt und dort auf die Verfassung schwört. In Spanien drohen Puigdemont allerdings die Festnahme und ein Strafprozess, da der Haftbefehl gegen inn dort weiter in Kraft ist.

\section{Die Verweigerung der provisorischen Akkreditierung}

Carles Puigdemont war am Wahlsonntag als Spitzenkandidat des Bündnisses „Lliures per Europa” (Frei für Europa) in Spanien ins EU-Parlament (EP) gewählt worden. Unmittelbar nach der Wahl entbrannte ein Streit um seine Personalie. Nach Angaben von Puigdemonts Anwalt Paul Bekaert verweigerte inm das Parlamentspräsidium die vorläufige Akkreditierung als EU-Abgeordneter sowie den Zutritt zum Parlament. Das Brisante ist: Laut Bekaert seien die übrigen spanischen Abgeordneten bereits akkreditiert gewesen. Nach heftiger Kritik an der Entscheidung des Parlamentspräsidiums, lenkte der Präsident des EP Tajani schließlich ein: Übereinstimmenden Medienberichten zufolge hob er die vorläufige Akkreditierung aller Spanischen Abgeordneten mit der Begründung auf, dass die amtlichen Endergebnisse der Europawahl in Spanien noch nicht veröffentlich seien. Dabei wird Tajani mit den Worten zitiert, man wolle ,jegliche Einmischung in einen nationalen Vorgang" vermeiden. 


\section{Die Gretchenfrage der Immunität}

Angesichts der Vorgänge in Brüssel drängt sich die Frage auf, warum der Streit um Puigdemont nun ausgerechnet in der Verweigerung seiner vorläufige Akkreditierung als EU-Abgeordneter kulminiert. Auf den ersten Blick scheint es sich um politische Schikane zu handeln. Dahinter steht aber auch die Frage nach dem rechtlichen Status Puigdemonts als EU-Abgeordneter. Als EU-Parlamentarier würde er nämlich Immunitätsschutz genießen. Streitbar ist dabei bereits, ob und welche Rechte eine vorläufige Akkreditierung in diesem Zusammenhang begründen. Unabhängig von dieser Frage, sprechen jedoch gute Gründe dafür, dass der Katalane bereits nach dem status quo Immunitätsschutz genießt.

\section{Die zeitliche Geltung der Immunität}

Der Immunitätsschutz der EU-Abgeordneten erstreckt sich nach dem Wortlaut von Art. 9 des Protokolls über Vorrechte und Befreiungen der Abgeordneten von 1965 (ProtVB) auf die „Dauer der Sitzungsperiode“. Bereits der EuGH stellte fest, dass dieser Begriff weit auszulegen ist und zumindest die gesamte Dauer des Mandats erfasst. Den Beginn bzw. den Erwerb des Mandats legt das Unionsrecht auf die erste Sitzung des Parlaments nach der Wahl fest (Art. 5 Direktwahlakt), also den 2. Juli 2019. Gleichwohl dürfen die gewählten Kandidaten bis dahin nicht schutzlos gestellt werden. Eine solche Auslegung des Art. 9 ProtVB wäre mit der Schutzrichtung der Immunität nicht zu vereinbaren. Denn die parlamentarische Immunität ist kein Privileg einzelner Mitglieder, sondern die Garantie für die Unabhängigkeit des Parlaments und seiner Mitglieder im institutionellen Gefüge der Europäischen Union. Die Unabhängigkeit des Parlaments ist aber nur dann gewährleistet, wenn auch der Erwerb eines Mandates seiner Mitglieder ohne politische Verfolgung möglich ist. Der Immunitätsschutz muss deshalb zeitlich bereits vor der ersten konstituierende Sitzung des Parlamentes nach der Wahl greifen. Nur so kann verhindert werden, dass der Immunitätsschutz der EU-Abgeordneten durch nationale Regierungen bereits an der Quelle beschnitten wird. Als zeitlicher Anknüpfungspunkt für den Beginn des Immunitätsschutzes der EU-Abgeordneten kann die für die Mitgliedstaaten verpflichtende Bekanntgabe der amtlichen Wahlergebnisse der Mitgliedstaaten an das Europäische Parlament dienen (Art. 12 Direktwahlakt). Ab diesem Zeitpunkt stehen die national gewählten Kandidaten als Anwärter auf die EU-Mandate auf europäischer Ebene fest. Zeitgleich wird damit die für die Mandatsträger bestehende Bedrohungslage begründet, vor der sie das Privileg der Immunität schützen soll. Schenkt man den entsprechenden Medienberichten Glauben, ist die amtliche Bekanntgabe der Wahlergebnisse durch den spanischen Staat bisher nicht erfolgt.

\section{Der Umfang der Immunität}

Vorausgesetzt die spanische Regierung übermittelt alsbald die amtlichen Wahlergebnisse an das Europäische Parlament, stellt sich anschließend die Frage, ob die Immunität Puigdemonts in Spanien vor einer gerichtlichen Verfolgung bzw. einer Inhaftierung schützt. Da der strafrechtliche Vorwurf gegen Puigdemont zeitlich vor seiner Anwartschaft auf ein EU-Mandat liegt und nicht in direktem 
Zusammenhang mit einer im Amt getätigten Meinungsäußerung steht, ist normativer Anknüpfungspunkt für seinen Immunitätsschutz Art. 9 ProtVB. Die Norm formuliert ein dichotomisches Schutzniveau: Der Umfang des Immunitätsschutzes der EUAbgeordneten ist davon abhängig, in welchem Mitgliedstaat die strafverfolgenden Behörden angesiedelt sind. Droht einem Abgeordneten aus einem anderen Mitgliedstaat, als dem, aus dessen nationalen Kontingent er gewählt wurde, eine strafrechtliche Verfolgung, kann er gegen die Verfolgung den Grundsatz der Immunität in Stellung bringen. Verbürgt ist dieser unionsweite autonome Immunitätsschutz in Art. 9 lit b. ProtVB. Dagegen ordnet Art. 9 lit a ProtVB an, dass den EU-Abgeordneten im „Hoheitsgebiet ihres eigenen Staates“ die Immunität zusteht, die den nationalen Parlamentsmitgliedern gewährt wird. Damit garantiert die letztgenannte Norm eine Gleichbehandlung von EU-Abgeordneten und nationalen Abgeordneten in Immunitätsfragen und wirkt nur gegenüber dem „eigenen Staat“. Die dichotomische Normstruktur geht auf das früher obligatorische Doppelmandat der EU-Abgeordneten zurück, die vormals aus der Mitte der nationalen Parlamente berufen wurden und sollte eine doppelte Privilegierung der Abgeordneten verhindern. Im Ergebnis führt die Regelung dazu, dass in den Mitgliedstaaten unterschiedliche Schutzniveaus in Immunitätsfragen gelten. Dabei kann der in den Herkunftsländern der Abgeordneten gewährte Immunitätsschutz hinter dem durch Art. 9 lit b. für den Bereich der übrigen Mitgliedstaaten eingeräumten Immunitätsschutz durchaus zurückbleiben. In Spanien genießt Puigdemont demnach den gleichen Immunitätsschutz wie ein nationaler spanischer Abgeordneter. Das spanische Immunitätssystem gewährleistet grundsätzlich die Redefreiheit der Abgeordneten sowie den Schutz vor Festnahme, Verwahrung und behördlicher Verfolgung. Es entspricht damit dem klassischen Immunitätsmodell, wie es aus dem deutschen Recht bekannt ist. Gegen eine Inhaftierung in Spanien kann Carles Puigdemont also den Grundsatz der Immunität in Stellung bringen.

Art. 71 der spanischen Verfassung garantiert den Abgeordneten dort den Schutz vor Festnahme, Verwahrung und behördlicher Verfolgung.

\section{Aufhebung der Immunität}

Für die spanische Regierung bestünde dann immer noch die Möglichkeit, die Aufhebung der Immunität Puigedemonts beim Europäischen Parlamentspräsidenten zu beantragen. Bei dem nach Art. 9 lit a ProtVB garantierten Immunitätsschutz wendet das EP in der Regel die nationalen Immunitäts- und Verfahrensvorschriften an. An die nationale Entscheidungspraxis ist es dabei allerdings nicht gebunden. Vielmehr räumt Art. 9 Abs. 3 ProtVB dem EP einen eigenen Entscheidungsspielraum ein. Durch seine Kandidatur als EU-Abgeordneter hat Puigdemont damit letztlich einen heiklen innerstaatlichen Konflikt zu einem europäischen Politikum gemacht. Ende offen.

Zitiervorschlag: Frederike Fründ, Carles Puigdemont und die Gretchenfrage der Immunität, JuWissBlog Nr. 62/2019 v. 13.6.2019, https://www.juwiss.de/62-2019/ 


\section{(c) (1) (9)}

Dieses Werk ist lizenziert unter einer Creative Commons Namensnennung - Nicht kommerziell - Keine Bearbeitungen 4.0 International Lizenz.

\section{(cc) BY-NC-ND}

